\title{
Mechanisms of Enamel Dissolution in Acid Buffers
}

WILLIAM I. HIGUCHI, ${ }^{*}$ JOHN A. GRAY,† JOHN J. HEFFERREN, $\ddagger$ and

PRAFULL R. PATEL*

A quantitative understanding of the mechanism of human dental enamel dissolution in buffers is important because it should lead to a clear description of the causative factors in caries formation, and it can be profitably employed in the design of meaningful screening methods for potential anti-decay agents.

Gray $^{1}$ has reported studies on enamel dissolution, concluding that the rates of dissolution of enamel in his buffers were controlled by the diffusion rates of the reactants and products of the dissolution reaction. He also pointed out that the initial enamel dissolution rate is largely a function of the total buffer concentration, buffer acid strength, and $\mathrm{pH}$. The effects of common and foreign ions on the rates of enamel dissolution were accounted for in a consistent fashion.

Gray deduced a semi-empirical equation for the rate of dissolution which rather satisfactorily describes all the experimental findings. This relationship includes the bufferacid dissociation constant, the total buffer concentration, the hydrogen-ion concentration, and other terms, when necessary, for common and foreign ions affecting the rate.

The success of this equation suggested that detailed mathematical models could be formulated and tested, employing simultaneous diffusion and chemical reaction, as has been done in describing the dissolution behavior of benzoic acid ${ }^{2}$ in basic and buffered media. The present communication is a report on the study of two such models. These have been examined with Gray's data. The analyses demonstrate the usefulness of this approach in future studies on dental problems.

This investigation was supported in part by USPHS Research Grant DE-01830-01 from the National Institute of Dental Research, National Institutes of Health, Bethesda, Md.

Received for publication August 29, 1963. Mich.

* College of Pharmacy, University of Michigan, Ann Arbor,

$\dagger$ Miami Valley Laboratories, Procter \& Gamble Co., Cincinnati, Ohio.

† Division of Dentistry, American Dental Association, Chicago, IIl.

\section{Materials and Methods}

General consideration.-As it is well known ${ }^{3}$ that the mineral portion of tooth enamel is mostly hydroxyapatite, the assumption that the surface of the enamel is hydroxyapatite during dissolution is a plausible one. The case based on this assumption will be called "Model A." As will be seen later, this model appears to provide the best description of enamel dissolution rate over most of Gray's data.

Because demineralization in the acid $\mathrm{pH}$ range is most interesting and because under equilibrium conditions dicalcium phosphate dihydrate is more stable than hydroxyapatite in aqueous acid media, it seemed that one should also examine the possibility that surface conversion of hydroxyapatite to dicalcium phosphate dihydrate may occur concomitantly with demineralization. A special case of this combination of processes will be called "Model B."

There are certainly other models that should be considered, but this paper was limited to the two models which seemed most reasonable and were mathematically tractable. Future experimental studies dictated by the present analysis of mechanisms may necessitate refinement of the present models or introduction of entirely new ones.

The two models, A and B, are illustrated in Figures 1 and 2. In both cases, when the enamel is exposed to an acid-buffer solution, the following processes take place. The acidbuffer molecules and hydrogen ions diffuse toward the surface across a liquid diffusion layer of constant thickness $h$. These acidic molecules and ions meet the $\mathrm{PO}_{4}^{3-}$ and/or the $\mathrm{HPO}_{4}^{2-}$ that are diffusing outward from the enamel surface through the liquid diffusion layer. Reaction occurs in the diffusion layer to the extent determined by the dissociation constants for the $\mathrm{HPO}_{4}^{2-}, \mathrm{H}_{2} \mathrm{PO}_{4}^{1--}$, and the buffer acid, as well as by the concentrations of all the species involved. The reac- 


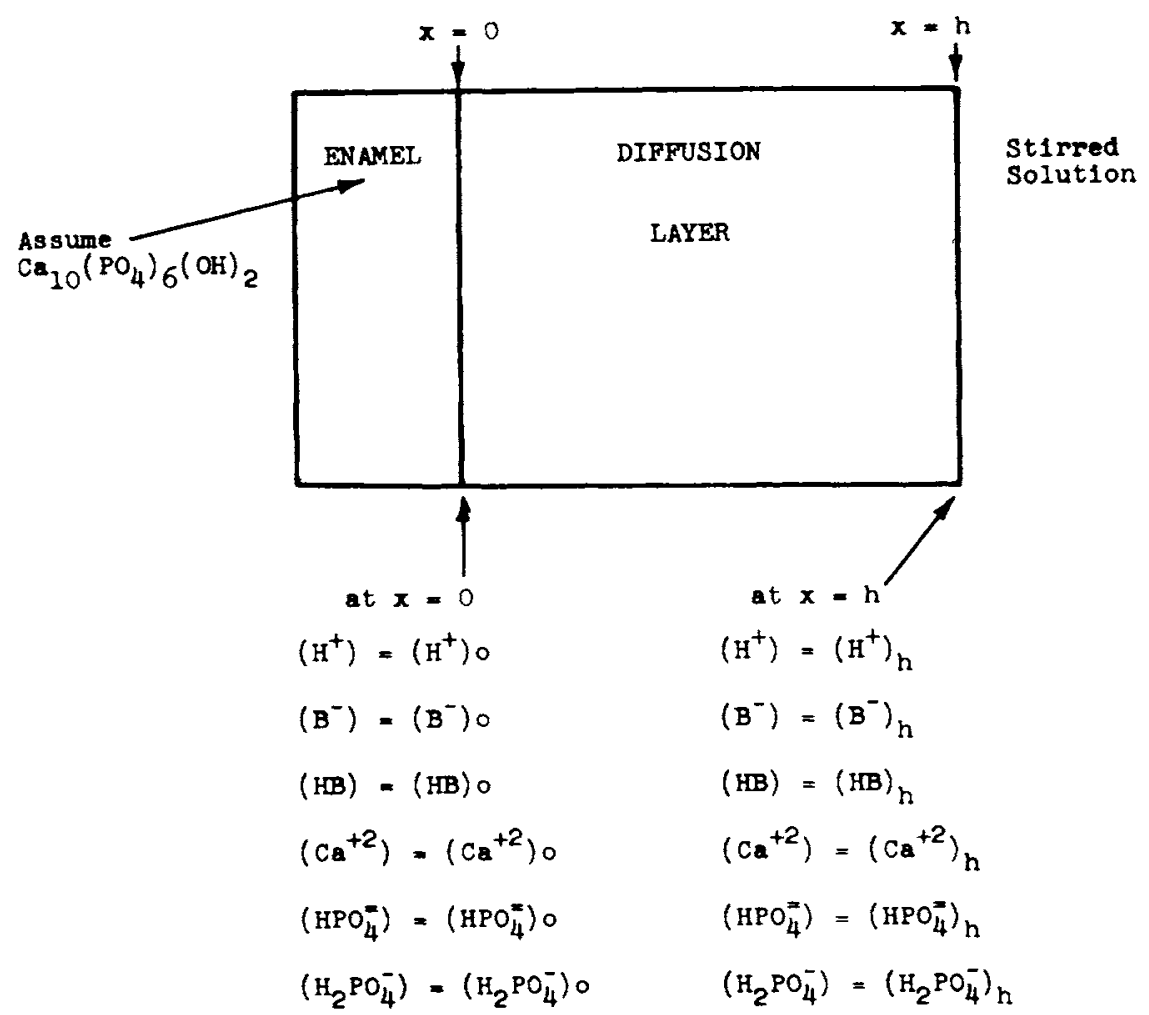

FIG. 1.-Model A. Dissolution of enamel in acid buffer solutions. $\left[\mathrm{H}^{+}\right],\left[\mathrm{Ca}^{+2}\right]$, etc., are the concentrations of the respective species. Solution adjacent to surface is in equilibrium with hydroxyapatite phase.

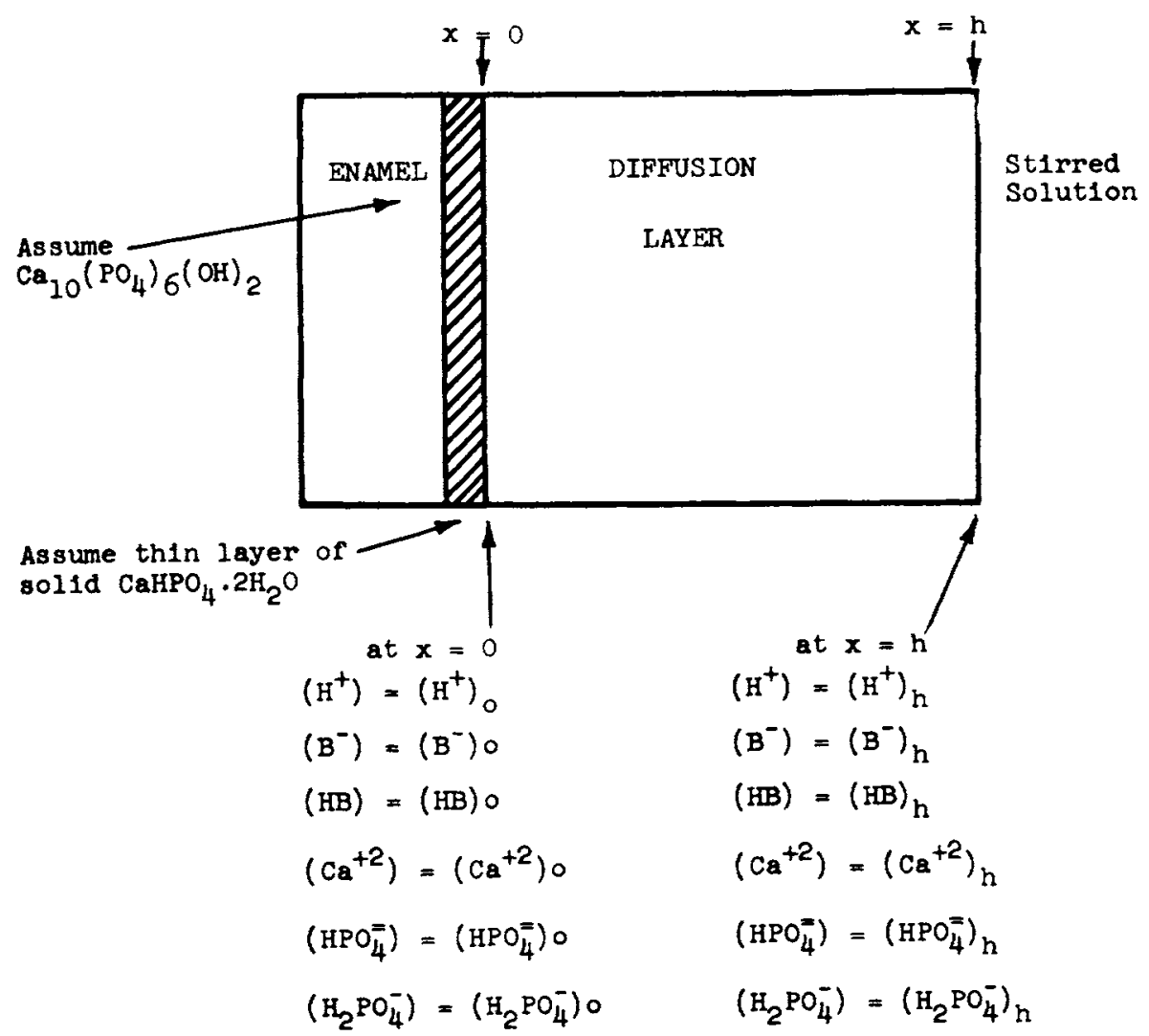

FIG. 2.--Model B. Dissolution of enamel in acid buffer solutions, assuming that solution adjacent to surface is in equilibrium with dicalcium phosphate dihydrate. 
tion products then diffuse out of the diffusion layer into the bulk solution along with the $\mathrm{Ca}^{2+}$ and remaining unreacted phosphate species. In "Model A," it is assumed that the enamel surface exposed to the solution is $\mathrm{Ca}_{10}\left(\mathrm{PO}_{4}\right)_{6}(\mathrm{OH})_{2}$ and that the solution immediately adjacent to the interface is in equilibrium with this phase. In "Model $B$," it is assumed that a thin layer of dicalcium phosphate dihydrate exists at the interface and that the solution adjacent to the surface is in equilibrium with this solid phase. As will be seen from the theoretical calculations, the tendency for conversion of hydroxyapatite to dicalcium phosphate becomes appreciable at relatively high rates of enamel dissolution. The appropriate equations can now be set up for each of the two models.

Model A (hydroxyapatite).--The following steady-state* equations describe the movement and the reactions of the species in the diffusion layer as discussed above.

$$
\begin{aligned}
D_{\mathrm{Ca}} \frac{d^{2}\left[\mathrm{Ca}^{2+}\right]}{d x^{2}} & =0 \\
D_{\mathrm{HP}} \frac{d^{2}\left[\mathrm{HP} 0_{4}^{=}\right]}{d x^{2}}-\phi_{1}-\phi_{2} & =0 \\
D_{\mathrm{H} 2 \mathrm{P}} \frac{d^{2}\left[\mathrm{H}_{2} \mathrm{P} 0_{4}^{-}\right]}{d x^{2}}+\phi_{1}+\phi_{2} & =0 \\
D_{\mathrm{H} \mathrm{B}} \frac{d^{2}[\mathrm{HB}]}{d x^{2}}-\phi_{2}+\phi_{3} & =0 \\
D_{\mathrm{B}} \frac{d^{2}\left[\mathrm{~B}^{-}\right]}{d x^{2}}+\phi_{2}-\phi_{3} & =0 \\
D_{\mathrm{H}} \frac{d^{2}\left[\mathrm{H}^{+}\right]}{d x^{2}}-\phi_{1}-\phi_{3} & =0
\end{aligned}
$$

These are expressions for Fick's law of diffusion taking into account the mass balances in the simultaneous diffusion and rapid chemical reaction. Because of the thinness of the diffusion layer, it is necessary to consider only one directional coordinate, $x$, which is perpendicular to the surface (see Figs. 1 and 2). The $D$ 's in equations (1-6) are the respective diffusion coefficients for $\mathrm{Ca}^{2+}, \mathrm{HPO}_{4}^{-}, \mathrm{H}_{2} \mathrm{PO}_{4}^{-}$, monobasic buffer acid molecule $\mathrm{HB}$, the buffer ion $\mathrm{B}^{-}$, and $\mathrm{H}^{+}$. The bracketed quantities $\left[\mathrm{Ca}^{2+}\right]$, $\left[\mathrm{HPO}_{4}^{-}\right]$, etc., are the concentrations of the species.

* It can easily be shown that the non-steady-state portion of this problem is of negligible importance.
The quantities $\phi_{1}, \phi_{2}$, and $\phi_{3}$ are, respectively, the rates of reaction per unit volume for the following reactions:

$$
\begin{aligned}
\mathrm{HPO}_{4}^{-}+\mathrm{H}^{+} & \rightarrow \mathrm{H}_{2} \mathrm{PO}_{4}^{-}, \\
\mathrm{HPO}_{4}^{-}+\mathrm{HB} & \rightarrow \mathrm{H}_{2} \mathrm{PO}_{4}^{-}+\mathrm{B}^{-}, \quad \text { and } \\
\mathrm{H}^{+}+\mathrm{B}^{-} & \rightarrow \mathrm{HB} .
\end{aligned}
$$

These reaction rates are, of course, assumed to be diffusion-controlled. If other reactions occur in the solution phase, equations (1-6) may not describe the situation entirely. Thus, for example, if the calcium-complexing agent is present, these equations may not suffice. In such cases, it may be necessary to revise the equations.

Along with equations (1-6), the following equilibrium expressions must apply at various places in the diffusion layer:

$$
\text { at } x>0, K_{\mathrm{B}}=\frac{\left[\mathrm{H}^{+}\right]\left[\mathrm{B}^{-}\right]}{[\mathrm{HB}]}
$$

$$
\text { at } x>0, K_{2 \mathrm{P}}=\frac{\left[\mathrm{H}^{+}\right]\left[\mathrm{HPO}_{4}^{=}\right]}{\left[\mathrm{H}_{2} \mathrm{PO}_{4}^{-}\right]} \text {; }
$$

$$
\text { at } x>0, K_{3 \mathrm{P}}=\frac{\left[\mathrm{H}^{+}\right]}{\left[\mathrm{HPO}_{4}^{-1}\right]} \text {; }
$$

at $x=0$,

$$
\begin{gathered}
K_{\mathrm{HAP}}=\left[\mathrm{Ca}^{2+}\right]_{o}^{10}\left[\mathrm{PO}_{4}^{3-}\right]_{o}^{6}\left[\mathrm{OH}^{-}\right]_{o}^{2} ; \\
\text { at } x>0, K_{w}=\left[\mathrm{H}^{+}\right]\left[\mathrm{OH}^{-}\right] .
\end{gathered}
$$

These $K$ expressions are the concentration equilibrium constants determining the relative concentrations of the species at any plane in the diffusion layer.

There are other boundary conditions which can be established. At $0 \leq x \leq h$ :

$$
-D_{\mathrm{HB}} \frac{d[\mathrm{HB}]}{d x}=D_{\mathrm{B}} \frac{d\left[\mathrm{~B}^{-}\right]}{d x} \text {. }
$$

Equation (12) means that the rate of $\mathrm{HB}$ reaching the enamel interface region must equal the amount of $\mathrm{B}^{-}$leaving the interface. Also, at $0 \leq x \leq h$ :

$$
\begin{aligned}
& 10\left\{D_{\mathrm{H}} \frac{d\left[\mathrm{H}^{+}\right]}{d x}+D_{\mathrm{H}_{2} \mathrm{P}} \frac{d\left[\mathrm{H}_{2} \mathrm{PO}_{4}^{-}\right]}{d x}\right. \\
& \left.+D_{\mathrm{H}} \frac{d[\mathrm{HB}]}{d x}\right\}=-8 D_{\mathrm{Ca}} \frac{d\left[\mathrm{Ca}^{2+}\right]}{d x}
\end{aligned}
$$


and

$$
\begin{gathered}
10\left\{D_{\mathrm{HP}} \frac{d\left[\mathrm{HPO}_{4}^{-}\right]}{d x}+D_{\mathrm{H} 2 \mathrm{P}} \frac{d\left[\mathrm{H}_{2} \mathrm{PO}_{4}^{-}\right]}{d x}\right\} \\
=6 D_{\mathrm{Ca}} \frac{d\left[\mathrm{Ca}^{2+}\right]}{d x} .
\end{gathered}
$$

Both equations (13) and (14) follow from the assumed net reaction:

$$
\begin{gathered}
\mathrm{Ca}_{10}\left(\mathrm{PO}_{4}\right)_{6}(\mathrm{OH})_{2}+8 \mathrm{H}^{+}=10 \mathrm{Ca}^{2+} \\
+6 \mathrm{HPO}_{4}^{=}+2 \mathrm{H}_{2} \mathrm{O}
\end{gathered}
$$

Equation (15) does not necessarily give the mechanism but merely expresses the net reaction. The particular phosphate species involved depend on the solutions to equations $(1-14)$.

Equations (1-6) can now be integrated using the conditions given by equations $(7-15)$. For this purpose it is necessary to assume that the diffusion coefficients and the equilibrium constants are independent of the position in the diffusion layer. This is a fairly good assumption for present purposes because the buffer acts as a swamping electrolyte to give essentially constant ionic strength in the diffusion layer in most cases. The first integration of equations (1-6) with consideration of equations (12-14) gives the following set of equations:

$$
\begin{aligned}
& D_{\mathrm{C} \Omega} \frac{d\left[\mathrm{Ca}^{2+}\right]}{d x}=C_{1} \text {; } \\
& D_{\mathrm{HP}} \frac{d\left[\mathrm{HPO}_{4}^{-}\right]}{a x}+D_{\mathrm{H} 2 \mathrm{P}} \frac{d\left[\mathrm{H}_{2} \mathrm{PO}_{4}^{-}\right]}{d x} \\
& =0.6 C_{1} \text {; } \\
& D_{\mathrm{H}} \frac{d[\mathrm{HB}]}{d x}+D_{\mathrm{B}} \frac{d\left[\mathrm{~B}^{-}\right]}{d x}=0 \text {; } \\
& D_{\mathrm{H} 2 \mathrm{P}} \frac{d\left[\mathrm{H}_{2} \mathrm{PO}_{4}^{-}\right]}{a x}+D_{\mathrm{H}} \frac{d\left[\mathrm{H}^{+}\right]}{d x} \\
& +D_{\mathrm{HB}} \frac{d[\mathrm{HB}]}{d x}=-0.8 C_{1} .
\end{aligned}
$$

Here, $C_{1}$ is a constant of integration. The other three constants of integration were eliminated by means of equations (12-14). From equation (16), it can be seen that, physically, $C_{1}$ is the negative of the rate of diffusion of $\mathrm{Ca}^{2+}$ through the layer. For convenience, let

$$
G=-C_{1},
$$

where $G$ is the diffusion rate of $\mathrm{Ca}^{2+}$ and is the quantity giving the rate of enamel dissolution.

Equations (16-19) are integrated again, and the symbols given in Figure 1 for the species concentration at $x=0$ and $x=h$ are substituted in the resulting expressions. This integration over the limits $x=0$ and $x=h$ gives:

$$
\begin{gathered}
D_{\mathrm{Ca}}\left(\left[\mathrm{Ca}^{2+}\right]_{h}-\left[\mathrm{Ca}^{2+}\right]_{o}\right)=-G h \\
D_{\mathrm{HP}}\left(\left[\mathrm{HPO}_{4}^{-=}\right]_{h}-\left[\mathrm{HPO}_{4}^{=}\right]_{o}\right)+D_{\mathrm{H}_{2} \mathrm{P}} \\
\times\left(\left[\mathrm{H}_{2} \mathrm{PO}_{4}^{-}\right]_{h}-\left[\mathrm{H}_{2} \mathrm{PO}_{4}^{-}\right]_{o}\right)=0.6 G h \\
D_{\mathrm{HB}}\left([\mathrm{HB}]_{h}-[\mathrm{HB}]_{o}\right)+D_{\mathrm{B}}\left(\left[B^{-}\right]_{h}\right. \\
\left.-\left[B^{-}\right]_{o}\right)=0 ; \\
D_{\mathrm{H}_{2} \mathrm{P}}\left(\left[\mathrm{H}_{2} \mathrm{PO}_{4}^{-}\right]_{h}-\left[\mathrm{H}_{2} \mathrm{PO}_{4}^{-}\right]_{o}\right)+D_{\mathrm{H}} \\
\quad \times\left(\left[\mathrm{H}^{+}\right]_{h}-\left[\mathrm{H}^{+}\right]_{o}\right)+D_{\mathrm{HB}} \\
\quad \times\left([\mathrm{HB}]_{h}-[\mathrm{HB}]_{o}\right)=0.8 G h
\end{gathered}
$$

If the product $G h$ is taken as a single unknown, it is possible, in principle, to eliminate $\left[\mathrm{Ca}^{2+}\right]_{o},\left[\mathrm{HPO}_{4}^{=}\right]_{o},\left[\mathrm{H}_{2} \mathrm{PO}_{4}^{-}\right]_{o},\left[\mathrm{~B}^{-}\right]_{0}$, $[\mathrm{HB}]_{o}$, and $\left[\mathrm{H}^{+}\right]_{o}$ from equations $(21-24)$ and equations $(7-11)$. In the present instance, because the situation is algebraically complicated, a single explicit equation for $G h$ is difficult to obtain. However, the entire set can be reduced to two equations. One of these results from the combination of equations (22), (23), (24), (7), and (8):

$$
\begin{aligned}
& \frac{0.6 G h+D_{\mathrm{HP}}\left[\mathrm{HPO}_{4}^{=}\right]_{h}+D_{\mathrm{H}_{2} \mathrm{P}}\left[\mathrm{H}_{2} \mathrm{PO}_{4}^{-}\right]_{h}}{D_{\mathrm{H} 2 \mathrm{P}}+\left(D_{\mathrm{HP}} K_{2 \mathrm{P}}\right) /\left(\left[\mathrm{H}^{+}\right]_{o}\right)} \\
& =\left[\mathrm{H}_{2} \mathrm{PO}_{4}^{-}\right]_{h}+\frac{D_{\mathrm{H}}}{D_{\mathrm{H} 2 \mathrm{P}}}\left[\mathrm{H}^{+}\right]_{h} \\
& -\frac{D_{\mathrm{H}}}{D_{\mathrm{H}: \mathrm{P}}}\left[\mathrm{H}^{+}\right]_{o}+\frac{D_{\mathrm{HB}}}{D_{\mathrm{H} 2 \mathrm{P}}}[\mathrm{HB}]_{h}^{-} \\
& -\frac{D_{\mathrm{HB}}}{D_{\mathrm{H} 2 \mathrm{P}}}\left\{\frac{D_{\mathrm{HB}}[\mathrm{HB}]_{h}+D_{\mathrm{B}}\left[\mathrm{B}^{-}\right]_{h}}{D_{\mathrm{HB}}+\left(D_{\mathrm{B}} K_{\mathrm{B}}\right) /\left(\left[\mathrm{H}^{+}\right]_{o}\right)}\right\} \\
& -\frac{0.8 G h}{D_{\mathrm{H} 2 \mathrm{P}}}
\end{aligned}
$$


The second of this pair of equations is obtained by combining equations $(8,9,10,11$, and 22):

$$
\begin{aligned}
& {\left[\mathrm{H}^{+}\right]_{o}^{14}=\frac{K_{3 \mathrm{P}}^{6} K_{w}^{2}}{K_{\mathrm{HAP}}}\left(\left[\mathrm{Ca}^{2+}\right]_{h}+\frac{G h}{D_{\mathrm{Ca}}}\right)^{10}} \\
& \left\{\frac{0.6 G h+D_{\mathrm{HP}}\left[\mathrm{HPO}_{4}^{=}\right]_{h}+D_{\mathrm{H}_{2} \mathrm{P}}\left[\mathrm{H}_{2} \mathrm{PO}_{4}^{-}\right]_{h}}{\left(D_{\mathrm{H} q \mathrm{P}}\right) /\left(K_{2 \mathrm{P}}\right)+\left(D_{\mathrm{HP}}\right) /\left(\left[\mathrm{H}^{+}\right]_{o}\right)}\right\}^{6} .
\end{aligned}
$$

Now $\left[\mathrm{H}^{+}\right]_{o}$ may be eliminated from equation (25) and equation (26), permitting evaluation of $G h$ for any set of values for the diffusion coefficients, equilibrium constants, and the species concentrations at $x=h$, all of which are either known or can be determined. These two are the working equations for the calculations to be discussed later.

Model B (Dicalcium Phosphate).Again, as in the case of "Model A," it is assumed that steady-state diffusion conditions are established in a negligible period of time. A further assumption is made that the thickness of the surface dicalcium phosphate dihydrate layer remains constant over the time range of interest. This means that the rate of formation of this layer must be equal to its rate of dissolution. Such a situation would be approximated by the case in which conversion takes place rapidly for those crystals near the enamel surface but more slowly for those crystals in the interior because of, for instance, slow diffusion of reactants in the enamel itself. No attempt beyond this will be made at the present to justify the physical plausibility of this model. Its primary value in the present report is that it provides another reasonable model that can be conveniently handled mathematically. The general case, i.e., where the dicalcium phosphate dihydrate layer thickness and density are permitted to vary, would be a more difficult problem to handle. However, such a problem may be worthwhile investigating in connection with the problem of the formation of "white spots." ${ }_{4,5}$

As Model B differs from Model A only in this surface boundary condition, all the equations for Model A will apply except equation (10) and, therefore, equation (26). In the present case,

$$
\text { at } x=0, K_{\mathrm{DCP}}=\left[\mathrm{Ca}^{2+}\right]_{o}\left[\mathrm{HPO}_{4}^{=}\right]_{o} \text {. }
$$

Therefore, instead of equation (26), we have for Model B:

$$
\begin{aligned}
& K_{\mathrm{DCP}}=\left(\left[\mathrm{Ca}^{2+}\right]_{h}+\frac{G h}{D_{\mathrm{Ca}}}\right) \\
& \left\{\frac{0.6 G h+D_{\mathrm{HP}}\left[\mathrm{HPO}_{4}^{-}\right]_{h}+D_{\mathrm{H} 2 \mathrm{P}}\left[\mathrm{H}_{2} \mathrm{PO}_{4}^{-}\right]_{h}}{D_{\mathrm{HP}}+\left(D_{\mathrm{H}_{2} \mathrm{P}}\left[\mathrm{H}^{+}\right]_{o}\right) /\left(K_{2 \mathrm{P}}\right)}\right\} .
\end{aligned}
$$

For Model B, then, equation (25) and equation (28) may be used to eliminate $\left[\mathrm{H}^{+}\right]_{o}$, and the resulting relation may be employed for the calculation of $G h$ for different conditions.

\section{Comparison of Theory with Data}

CONSIDERATIONS IN THE SELECTION OF CONSTANTS FOR THEORETICAL CALCULATrons.- The main problem in applying the above theoretical equations is that of selecting the values for all the constants appropriate for the conditions in Gray's studies. Except for the solubility product of hydroxyapatite, the values for most of the thermodynamic* equilibrium constants are known quite accurately. However, the values for these constants at the various ionic strengths encountered in Gray's experiments are difficult to estimate accurately even when specific interactions, e.g., complexing, are assumed to be unimportant. Another difficulty is the wide range of reported values for the thermodynamic solubility product for hydroxyapatite, $K_{\text {HAP }}$. Values appear to range from $1 \times 10^{-110}$ to $1 \times 10^{-120}$ even within a given sample. ${ }^{6}$ This corresponds to a spread of about a factor of three in molar solubility.

In view of these uncertainties, it was decided to make calculations in some cases with more than one set of values for the constants. In column 1 of Table 1 is given a set of values used in one series of calculations. These, except for the lactic acid dissociation constant, are based on values suggested by Brown ${ }^{7}$ for the thermodynamic dissociation constants. The thermodynamic lactic acid value here is the commonly accepted one. ${ }^{8}$

Another set of values for the constants is given in column 2 of Table 1 . These are the thermodynamic values corrected for ionic strength effects with the appropriate activity coefficients. Except for the $K_{\text {HAP }}$ value,

* Infinite dilution values, i.e., activity coefficient $=$ unity. 
the thermodynamic constants were essentially the same as those used in column 1 or taken from the tabulations of Stokes and Robinson. ${ }^{8}$ The value of $1 \times 10^{-120}$ was used here for the thermodynamic $K_{\text {HAP. }}$. This differs from the value of $1.3 \times 10^{-111}$ used for $K_{\mathrm{HAP}}$ in column 1 . The effects of using such different values of $K_{\text {HAP }}$ will be discussed later. With the exception of those for $\mathrm{Ca}^{2+}, \mathrm{HPO}_{4}^{2-}$, and $\mathrm{H}_{2} \mathrm{PO}_{4}^{-}$, in which cases Levinskas' values ${ }^{9,10}$ were used, the activity coefficients* were estimated from Kielland's tables. ${ }^{11}$ The values given in Table 1 are for $25^{\circ} \mathrm{C}$. Most of Gray's data were taken at $37^{\circ} \mathrm{C}$. It was decided that estimations of the temperature corrections were unwarranted in view of the uncertainties in the values of the constants. Furthermore, the data indicate only a slight temperature effect (about 20 per cent rate increase for a $10^{\circ}$ temperature rise), most of which should cancel when relative rates are under consideration, as they are here.

The value of $1 \times 10^{-5} \mathrm{~cm}^{2}$ per second was used for all diffusion coefficients. With perhaps the exception of the diffusion involving $\mathrm{H}^{+}$and $\mathrm{OH}^{-}$, this should be a reasonably good approximation $f$ for the molecules and ions involved here, and it can easily be shown that the diffusion of neither $\mathrm{H}^{+}$nor $\mathrm{OH}^{-}$is important over the range of conditions under present consideration.

Results of Calculations with Model A.- In Figure 3 the experimental data and the results of the theoretical calculations with equation (25) and equation (26) are presented for the initial dissolution rate of enamel in lactate buffer. The smooth curves are theory predictions based on the column-1 constants of Table 1. Column-2 values were employed for the broken curves. As the theory gives only the product $G h$, it was necessary to select the $h$ values which would provide a good fitf to theory of the data in each of the two cases. A value of $h=28 \mu$ was found to give the fit shown by

* The concept of individual activity coefficient is useful in estimating the activity coefficients of monovalent ions up to an ionic strength of about 0.1 . For divalent ions such as $\mathrm{Ca}^{2+}$ and HPO ${ }^{-}$, this practice may be subject to some question.

$\uparrow$ About \pm 50 per cent. See References 8 and 12 for some typical values of diffusion coefficients of molecules and ions in water.

\$ The procedure for obtaining a fit between data and theory (one model, one set of constants) essentially involves equating experiment to theory at some one point (one buffer concentration, one $\mathrm{pH}$ ). This then fixes the value for $h$. How well the other points compare with theory, then, is the test of the model. the smooth curves. A value of $h=16 \mu$ gave the fit shown by the broken curves. These values for $h$ are physically reasonable, as the thickness of most diffusion layers over a wide range of agitation conditions appears to be somewhere between 10 and $50 \mu .^{2,13}$

With both sets of constants, the comparison of data with theory is relatively satisfactory. It appears from these data alone that the theory for the rates may be insensitive to the selection of the values for constants. This is probably true to the extent that some of the activity coefficient effects cancel and some are absorbed in the adjustable unknown $h$-value. In future experimental studies, $h$ should be independently deter-

TABLE 1

EOUILIBRIUM CONSTANTS FOR THEORETICAL CALCULATIONS

\begin{tabular}{|c|c|c|}
\hline Parameter & $\begin{array}{l}\text { Column 1* } \\
\text { (Moles } \\
\left.\text { liter }{ }^{-1}\right)\end{array}$ & $\begin{array}{c}\text { Column 2† } \\
\text { (Moles } \\
\text { liter-1) }\end{array}$ \\
\hline $\begin{array}{l}K_{2 \mathrm{P}} \ldots \\
\left(K_{3 \mathrm{P}}^{6} K_{w}^{2}\right) /\left(K_{\mathrm{HAP}}\right) \\
K_{\mathrm{DCP}} \ldots \ldots \\
K_{\mathrm{B}} \text { (lactic) } \ldots \\
K_{\mathrm{B}} \text { (acetic) } \ldots \\
K_{\mathrm{B}} \text { (chloroacetic) }\end{array}$ & $\begin{array}{l}6.33 \times 10^{-8} \\
8.90 \times 10^{8} \\
2.77 \times 10^{-7} \\
1.39 \times 10^{-4} \\
\cdots \ldots \ldots \ldots \\
\ldots \ldots \ldots \ldots\end{array}$ & $\begin{array}{l}2.0 \times 10^{-7} \\
1.6 \times 10^{11} \\
3.0 \times 10^{-6} \\
2.0 \times 10^{-4} \\
2.5 \times 10^{-5} \\
1.9 \times 10^{-3}\end{array}$ \\
\hline
\end{tabular}

* Thermodynamic values, activity coefficient $=$ unity.

$\dagger$ Constants estimated for ionic strength $=0.1-0.2$.

mined by measuring dissolution rates of compounds with known diffusion coefficients under identical geometric and hydrodynamic conditions.

The dependence of rate on both the $\mathrm{pH}$ and buffer concentration at the lower buffer concentrations agrees very satisfactorily with theory, employing either set of values for the constants. At the higher buffer concentration, the theoretical results are generally lower than the experimental results. If this model is correct, these deviations may result from the smaller activity coefficients of ions at higher ionic strengths. If the activity coefficients of $\mathrm{Ca}^{2+}$ and $\mathrm{HPO}_{4}^{2-}$ were about $25-50$ per cent smaller at the higher buffer concentrations, the agreement between data and theory could be shown to be much better over the entire range, except for the single data point at $\mathrm{pH}=4$ and $[\mathrm{TB}]=$ $0.6 \mathrm{M}$. Such changes of this order of magni- 
tude in activity coefficients are possible, as can be seen in tables of mean activity coefficients. ${ }^{8}$

The possible importance of complex formation between $\mathrm{Ca}^{2+}$ and the lactate ion should be considered. At an ionic strength of about 0.2 , the dissociation constant for this one-to-one complex is about $0.1{ }^{14}$ The presence of this complexing tendency should result in an increase over the results in Figure 3 in dissolution rate, with increase in lactate buffer concentration at constant $\mathrm{pH}$ or with increasing $\mathrm{pH}$ at constant buffer concentration. The estimated magnitude of this effect and the predicted trends are also consistent with the observed deviations of the theoretical calculations from the data. To make such calculations, it is necessary to modify equation (21) and equation (23). The diffusion of the complex must be incorporated into equa- tion (21), and another term must be added to the mass-balance equation (23). In an experimental program with synthetic calcium phosphate phases, presently in progress, these points will be examined in greater detail.

It is worthwhile to mention that taking values for the constants somewhat larger or smaller than those given in Table 1 does not significantly improve the fit of the data to theory. These other calculations show that, to within about a factor of 2 or so, the value $1 \times 10^{-5} \mathrm{~cm}^{2}$ second $^{-1}$ for the diffusion coefficients appears to give the best fits when used with the other values in Table 1 . If a much larger or much smaller value for the diffusion coefficient is used, the fit is significantly poorer. Other calculations also show that, to within about a factor of $1 \times 10^{5}$, the values for $\left(K_{3 \mathrm{P}}^{6} K_{w}^{2}\right) /\left(K_{\mathrm{HAP}}\right)$ given in Table

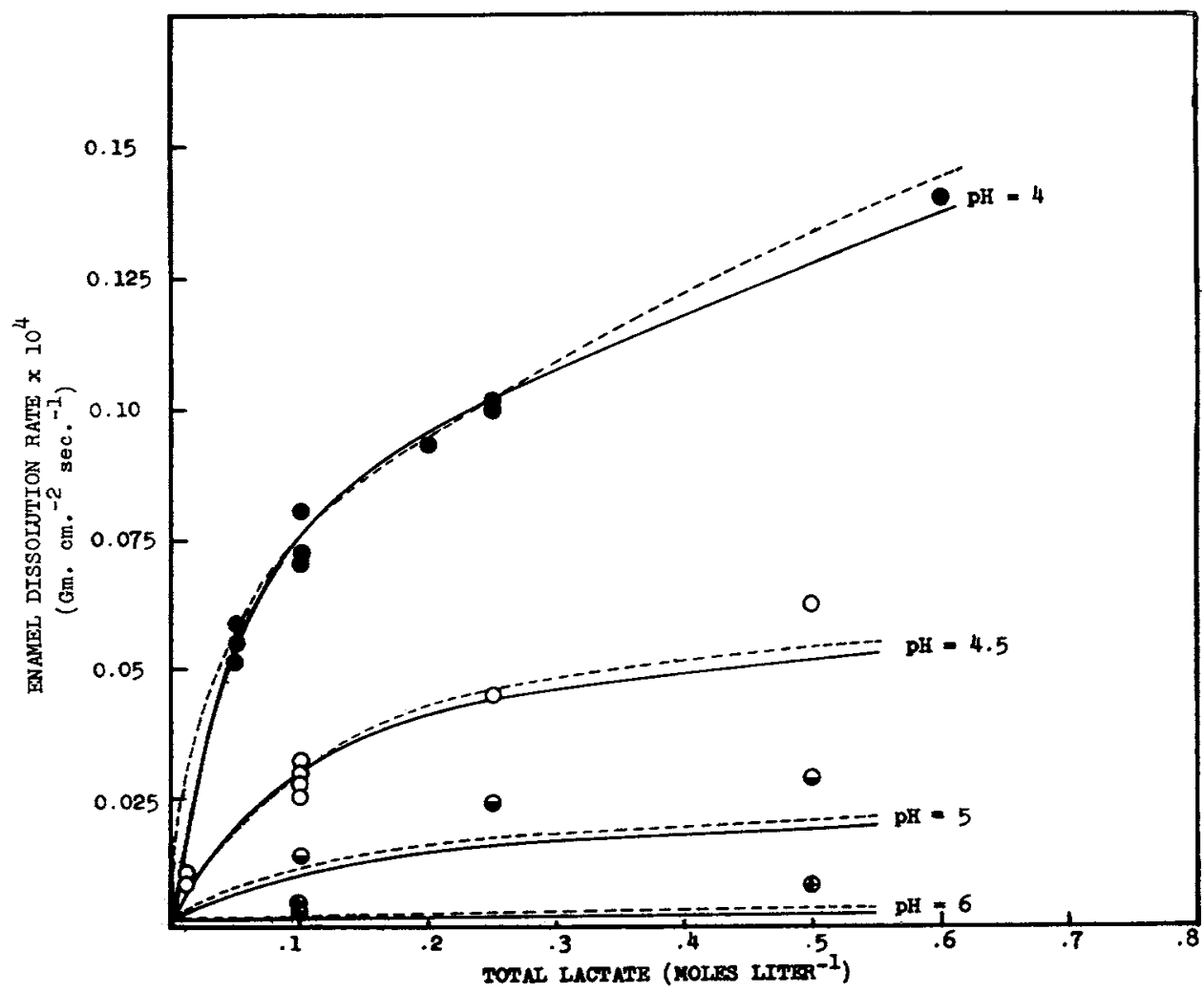

FIG. 3.- Initial dissolution rates of enamel in lactic acid buffers. Smooth curves correspond to Model A theory with $h=28 \mu$ and the column-1 values of Table 1 for the constants. Broken curves correspond to Model $A$ theory with $h=16 \mu$ and the column-2 values of Table 1 for the constants. The four sets of points are the experimental values at the four different $\mathrm{pH}$ conditions. 
1 give the best fits when used with the other values in their respective sets.

In Figures 4 and 5, the experiments and the Model A theory (the "A" curves) for the common ion effects are compared. The A-1 and the smooth A-2 curves correspond to the column-1 and column-2 values for the constants. The A-2 broken curves correspond to theory under the same conditions as the A-2 smooth curves except that the value for $\left(K_{3 \mathrm{~F}}^{6} K_{w}^{2}\right) /\left(K_{\mathrm{HAP}}\right)$ was taken $1 \times 10^{4}$ times larger (or what is the same, $K_{\mathrm{HAP}}$ was taken $1 \times 10^{4}$ times smaller). A similar improvement in the results of the A-1 curves would also be expected by taking a somewhat larger value for the corresponding $\left(K_{3 \mathrm{P}}^{6} K_{w}^{2}\right) /$ $\left(K_{\mathrm{HAP}}\right)$. In view of the uncertainties in the thermodynamic value ${ }^{6}$ for $K_{\mathrm{HAP}}$, in view of the uncertainty in the data-fitting process, and in view of the experimental deviations themselves, the observed common ion effects appear to be reasonably consistent with the Model A theory.

Another look at Model $\mathrm{A}$ is made possible by a comparison of the theory with data obtained with other acid buffers. Here calculations were carried out using only the values from column 2 of Table 1 for the constants. In Table 2 the experimental and Model A theoretical results are compared in columns 4 and 5. Again, the theoretical values were calculated using equation (25) and equation (26) with the previously established $h$-value of $16 \mu$. There is general agreement between experiment and theory (Model A), giving additional strength to this theory.

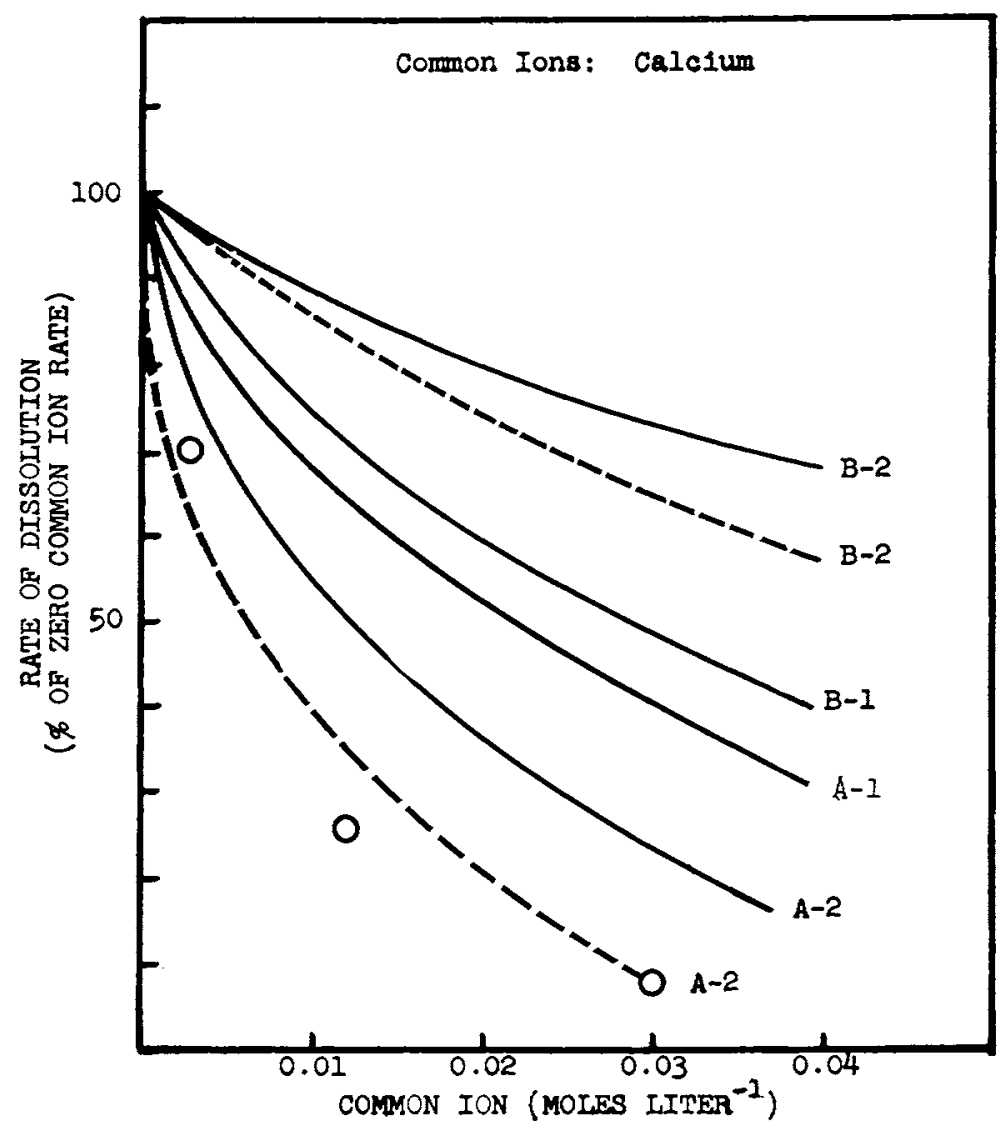

FIG. 4.-Effects of the common ion, $\mathrm{Ca}^{2+}$, on the initial dissolution rate of enamel at $\mathrm{pH}=4.5$ and total buffer concentration $=0.1 \mathrm{M}$. Circles are experimental data. Curves are theory, the letter referring to model and the number to either the column-1 or column-2 values in Table 1 for the constants. See text for more explanation. 
Results of Calculations with Model B.-Figure 6 shows the theoretical results obtained with equation (25) and equation (28), employing the constants given in columns 1 and 2, respectively, of Table 1 . These are compared with the experimental initial dissolution rates in lactate buffer. For the fits of data to theory as shown, a value of $h=28 \mu$ was taken for the smooth curves (column-1 constants), and a value of $h=29 \mu$ was taken for the broken curves (column-2 constants). It is apparent that a somewhat better fit for the data at $\mathrm{pH}$ values of 4.5 and 5.0 would be obtained in the case of the calculations with the column 1 constants if a larger $h$-value were used. This would be at the expense of a poorer fit for the curve at
$\mathrm{pH} 6.0$ and the higher rate points in the curve at $\mathrm{pH} 4.0$. The general agreement of data with Model B employing the values in column 2 is remarkably good. The only significant deviations in this instance occur at the low buffer concentration region of the curve at $\mathrm{pH} 4.0$.

The " $\mathrm{B}$ " curves in Figures 4 and 5 give the results of Model B common ion effects using values for the constants from columns 1 and 2, respectively, of Table 1 . The B-2 broken curves correspond to the same conditions as the B-2 smooth curves but with $K_{\mathrm{DCP}}=1.5 \times 10^{-6}$. The agreement between experiment and theory is much poorer for Model B than for Model A. For the $\mathrm{Ca}^{2+}$ effects, the disagreement appears to be much

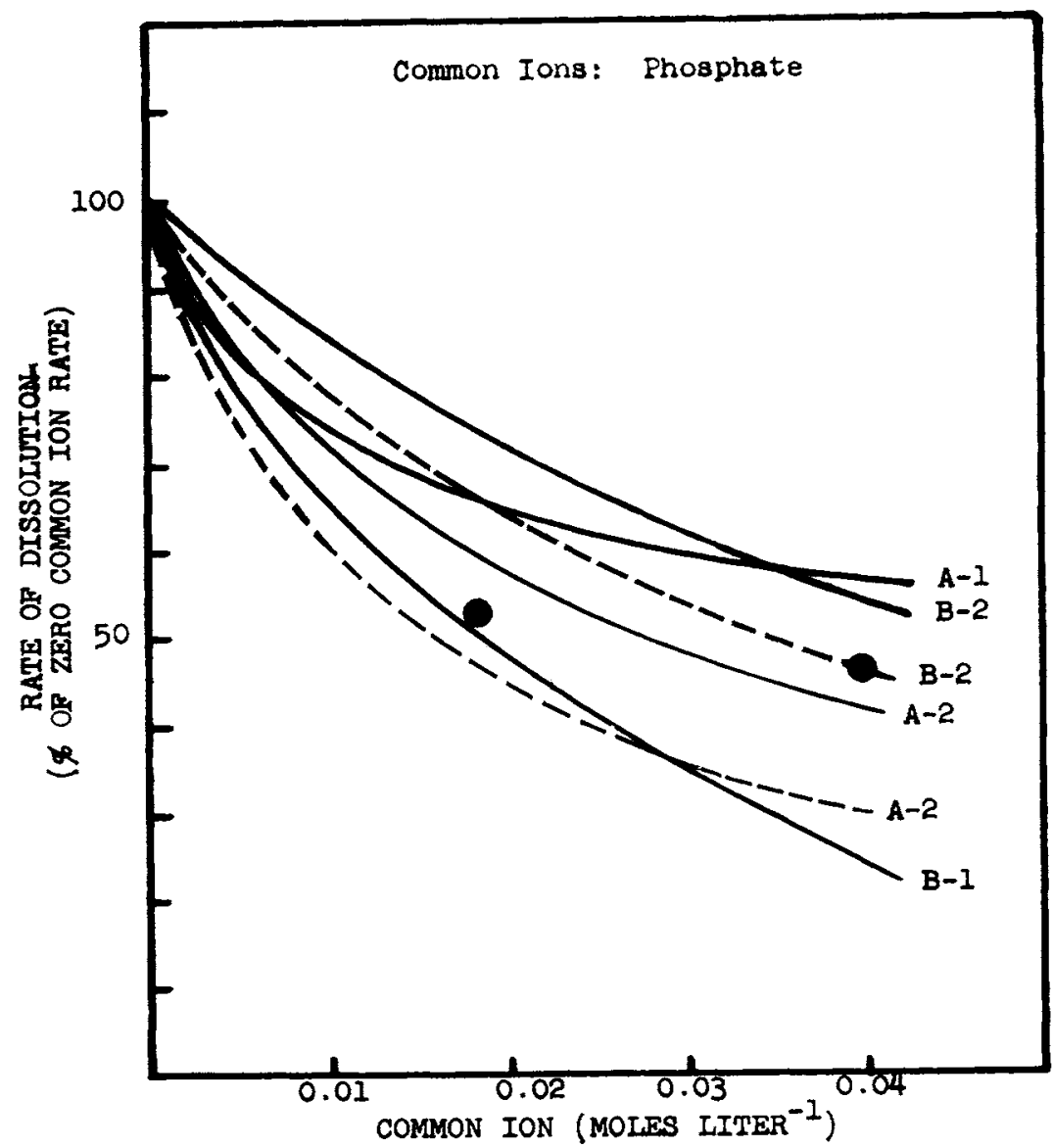

FIG. 5.-Effects of the common ion, phosphate, on the initial dissolution rate of enamel at $\mathrm{pH}=4.5$ and total buffer concentration $=0.1 \mathrm{M}$. Circles represent data. Curves, as in Fig. 4 , give the theoretical predicted rates. See text for more explanation. 
TABLE 2

ENAMEL Dissolution RATE in DIFFERENT BuFfERS AT PH 4.0

\begin{tabular}{|c|c|c|c|c|c|}
\hline Acid & $K_{\mathrm{n}}$ & (TB) & $\begin{array}{l}\text { Exp. } \\
\text { Rate }\end{array}$ & $\begin{array}{c}\text { Model A* } \\
\text { Rate }\end{array}$ & $\begin{array}{c}\text { Model } \mathrm{B} \dagger \\
\text { Rate }\end{array}$ \\
\hline $\begin{array}{l}\text { Acetic . . . . . . . . . . . . } \\
\text { Lactic . . . } \\
\text { Chloroacetic.... }\end{array}$ & $\begin{array}{l}2.5 \times 10^{-5} \\
2.0 \times 10^{-4} \\
1.9 \times 10^{-3}\end{array}$ & $\begin{array}{c}0.047 \\
.10 \\
0.60\end{array}$ & $\begin{array}{r}0.053 \\
.073 \\
0.108\end{array}$ & $\begin{array}{r}0.057 \\
.076 \\
0.087\end{array}$ & $\begin{array}{r}0.060 \\
.065 \\
0.067\end{array}$ \\
\hline
\end{tabular}

* For Model A calculations, $h=16 \mu$.

† For Model B calculations, $h=29 \mu$.

greater than could be explained on the basis of uncertainties in the values for the constants. Furthermore, Model B predicts that phosphate is more effective than $\mathrm{Ca}^{2+}$. This contradicts experimental results.

The last column of Table 2 gives the
Model B-calculated rates in other acid buffers. Constants in column 2 of Table 1 and equation (25) and equation (28) were used with $h=29 \mu$. It is apparent that Model B predictions are considerably poorer than those for Model A.

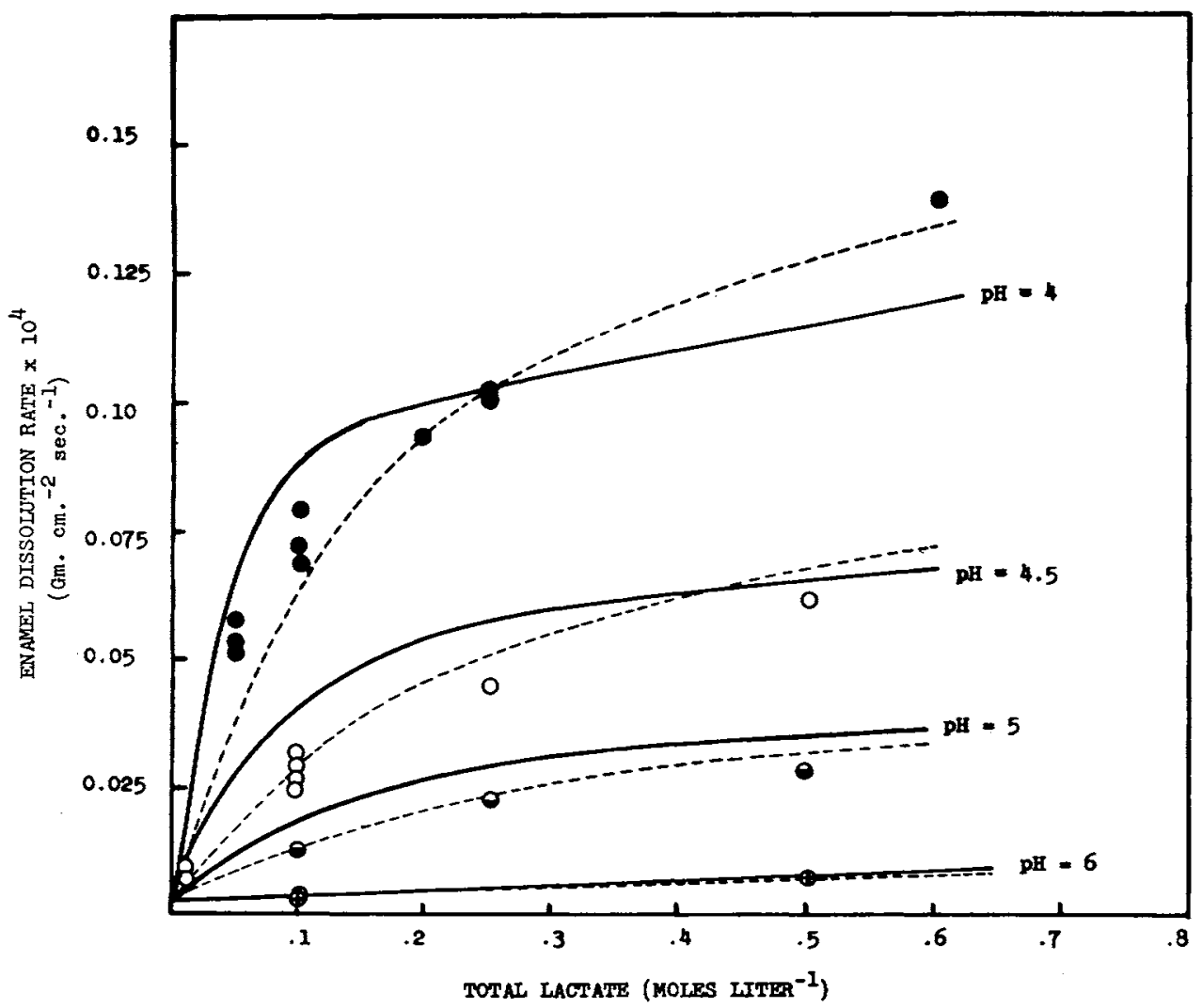

FIG. 6.-Initial dissolution rates of enamel in lactic acid buffers. Smooth curves correspond to Model B with $h=28 \mu$ and the column-1 values in Table 1 for the constants. Broken curves correspond to Model $\mathrm{B}$ with $h=29 \mu$ and the column-2 values. The four sets of points are the experimental data at the four $\mathrm{pH}$ conditions. 
TABLE 3

PRODUCT $\left[\mathrm{Ca}^{2+}\right]_{0}\left[\mathrm{HPO}_{4}\right]_{0}$ CALCULATED From MODEL A ${ }^{*}$

\begin{tabular}{|c|c|c|c|c|}
\hline $\begin{array}{c}G \\
\left(\mathrm{gm} \cdot \mathrm{cm}^{-2} \mathrm{sec} \cdot{ }^{-1}\right)\end{array}$ & {$\left[\mathrm{Ca}^{2+}\right]_{o}$} & {$\left[\mathrm{HPO}_{4}\right]_{o}$} & {$\left[\mathrm{Ca}^{2+}\right]_{o}\left[\mathrm{HPO}_{4}^{=}\right]_{o}$} & $K_{\mathrm{DCP}}$ \\
\hline $\begin{array}{l}1.45 \times 10^{-5} \ldots \ldots \\
5.5 \times 10^{-8} \cdots \cdots \\
2.5 \times 10^{-7} \ldots \ldots\end{array}$ & $\begin{array}{l}2.3 \times 10^{-2} \\
8.7 \times 10^{-3} \\
2.4 \times 10^{-4}\end{array}$ & $\begin{array}{r}3.1 \times 10^{-5} \\
3.7 \times 10^{-5} \\
5 \times 10^{-5}\end{array}$ & $\begin{array}{r}7 \times 10^{-7} \\
3.2 \times 10^{-7} \\
1.2 \times 10^{-8}\end{array}$ & $\begin{array}{l}3 \times 10^{-6} \\
3 \times 10^{-6} \\
3 \times 10^{-6}\end{array}$ \\
\hline
\end{tabular}

* Using equation (25) and equation (26) with constants from column 2 of Table 1.

TABLE 4

PRODUCT $\left(\left[\mathrm{Ca}^{2+}\right]_{a}^{\mathrm{pt}}\left[\mathrm{HPO}_{4}^{=}\right]_{0}^{14}\right) /\left(\left[\mathrm{H}_{2} \mathrm{PO}_{4}^{=}\right]_{0}^{8}\right)$ CALCULATED FROM MODEL B*

\begin{tabular}{|c|c|c|c|c|c|}
\hline $\begin{array}{c}G \\
\left.\text { (gm. cm. } .^{-2} \mathrm{sec}^{-1}\right)\end{array}$ & {$\left[\mathrm{Ca}^{2+}\right]_{0}$} & {$\left[\mathrm{HPO}_{4}^{\overline{-}}\right]_{0}$} & {$\left[\mathrm{H}_{2} \mathrm{PO}_{4}^{-}\right]_{0}$} & $\begin{array}{c}\left(\left[\mathrm{Ca}^{2+}\right]_{0}^{10}\left[\mathrm{HPO} \mathrm{O}_{\overline{4}}^{\overline{1}}\right]_{\bullet}^{1 \mathbf{4}}\right) / \\
\left(\left[\mathrm{H}_{2} \mathrm{PO}_{4}^{-}\right]_{a}^{8}\right)\end{array}$ & $\begin{array}{c}\left(K_{\mathrm{HAP}} K_{2 \mathrm{P}}^{8}\right) / \\
\left(K_{3 \mathrm{P}}^{6} K_{w}^{2}\right)\end{array}$ \\
\hline $\begin{array}{l}1.4 \times 10^{-5} \\
7 \times 10^{-6} \ldots \\
1.0 \times 10^{-6}\end{array}$ & $\begin{array}{l}4 \times 10^{-2} \\
2 \times 10^{-2} \\
3 \times 10^{-3}\end{array}$ & $\begin{array}{l}7.5 \times 10^{-5} \\
1.5 \times 10^{-4} \\
1.0 \times 10^{-3}\end{array}$ & $\begin{array}{l}2.4 \times 10^{-2} \\
1.2 \times 10^{-2} \\
8 \times 10^{-4}\end{array}$ & $\begin{array}{r}8 \times 10^{-60} \\
6.5 \times 10^{-56} \\
3.3 \times 10^{-43}\end{array}$ & $\begin{array}{l}1.5 \times 10^{-65} \\
1.5 \times 10^{-65} \\
1.5 \times 10^{-65}\end{array}$ \\
\hline
\end{tabular}

* Using equation (25) and equation (28) with constants from column 2 of Table 1.

\section{Discussion}

In the preceding section, it has been shown that from a rough quantitative point of view both Models A and B appear to be consistent with the general behavior of dental enamel in acid buffers. This, at first thought, may suggest that the present method of analyzing data may not be very critical with regard to distinguishing phases that are dissolving. In the present instance, this is partly true because the analysis is being applied to conditions that are very close to those corresponding to equilibrium between dicalcium phosphate dihydrate and hydroxyapatite. At the same time, the analysis suggests that, because the two models give similar predictions in many instances, the likelihood of a surface phase conversion of hydroxyapatite to dicalcium phosphate dihydrate is not entirely remote.

A closer examination of the two models has shown that Model A is much nearer to the experimental facts. The lack of quantitative agreement between the observed common ion effects and theory is perhaps the most significant inconsistency in the Model $\mathrm{B}$ situations. Also, the expected ionic strength effects and/or the calcium complexing effects appear to be present as deviations in the correct directions for Model A. Finally, Model A agrees better with data on other acid buffers.
A final examination of this problem is presented in Tables 3 and 4 . In Table 3 , the values for the product $\left[\mathrm{Ca}^{2+}\right]_{0}\left[\mathrm{HPO}_{4}^{-}\right]_{o}$ are given. These values were calculated with the Model A equations and the column-1 constants of Table 1. It can be seen that over the entire range of dissolution rates, the product is always less than the value $K_{\text {DCP }}=3 \times 10^{-6}$. This means that the surface is never supersaturated with respect to dicalcium phosphate, if Model $\mathrm{A}$ and the column-2 values are correct. However, it should be recalled that this discussion refers to initial dissolution rate conditions, and the existence of dicalcium phosphate has been demonstrated at equilibrium between acid and enamel. ${ }^{5}$ Table 3 shows that if Model B and the column-2 values are correct, the interface will always be supersaturated with respect to hydroxyapatite over the entire range of rates. As this then raises the question why the surface would convert to dicalcium phosphate in the first place, again Model B appears to fail.

If, however, a smaller value for $\left(K_{3 \mathrm{P}}^{6} K_{w}^{2}\right) /$ $\left(K_{\mathrm{HAP}}\right)$ is used in the calculations of Table 3 and Table 4 , the above conclusions may not apply entirely. Thus, for example, if $K_{\text {HAP }}=1 \times 10^{-111}$ is used instead of $K_{\text {HAP }}=1 \times 10^{-120}$ in the column-2 values, ${ }^{*}$

* This of course, as pointed out above, would lead to a poorer comparison of the theoretical common ion effect with data. 
one would find that calculations similar to those given in Tables 3 and 4 would predict with Model $\mathrm{A}$ that, at the very high initial rates ( $\mathrm{pH}=4$, high buffer concentrations), the surface would become supersaturated with respect to dicalcium phosphate dihydrate. In this situation, Model B may apply if the conversion rate is rapid enough. It must be kept in mind, however, that even if Model B becomes the thermodynamically favored one according to this diffusion-controlled theory, the initial rate of dissolution may yet obey Model A because of the possible slowness of the conversion rate. These are points that require further exploration, both experimentally and theoretically.

Future work would involve extending this method to more complex situations. Not only initial rates but integral rate problems could be examined as data became available. For situations where solution complexing may be important, the equations could be modified to accommodate the transport of the complex. Where new phases are suspected, e.g., where tin- or fluoridecontaining phases appear, the appropriate boundary conditions must be used with the differential equations. It should be possible to extend the technique to the problem of subsurface decalcification. 4,5 Answers to questions concerning mechanisms in this instance would be of extreme importance to the basic understanding of caries formation.

\section{Summary}

A method of analyzing enamel dissolution data has been presented. It is based on the consideration of simultaneous diffusion and chemical reactions of the various ionic and molecular species involved. The technique permits a critical test of examination of various physical models with experimental data. The method has been applied to Gray's data on the initial rates of enamel dissolution in acidic buffers. The results of the analyses of two models show that a solution diffusion-controlled mechanism, involving dissolution from a hydroxyapatite surface in equilibrium with the solution ad- jacent to the surface, best explains all the data. Both $\mathrm{pH}$ effects and buffer-concentration effects are quantitatively in agreement with this model. Common ion effects are also satisfactorily accounted for, and rate determinations in several acidic buffers agree well with the theory. At very high initial rates of dissolution, the theory indicates that conversion to dicalcium phosphate may become possible. That conversion actually takes place remains uncertain because of the uncertainties in the values of the parameters used in the theoretical calculations.

\section{References}

1. GRAY, J. A. Kinetics of the Dissolution of Human Dental Enamel in Acid, $J$. dent. Res., 41:633-45, 1962.

2. Higuchi, W. I., Parrott, E. L., Wurster, D. E., and Higuchi, T. Investigation of Drug Release from Solids. II. Theoretical and Experimental Study of Influences of Bases and Buffers on Rates of Dissolution of Acidic Solids, J. Amer. pharm. Ass. sci. Ed., 47: 376-83, 1958.

3. Posner, A. S. The Nature of the Inorganic Phase in Calcified Tissues. In Calcification in Biological Systems, ed. R. F. SognNaEs, pp. 373-94. (Publication No. 64.) Washington, D.C.: American Association for the Advancement of Science, 1960.

4. Gray, J. A., Francis, M. D., and Griebstein, W. J. Chemistry of Enamel Dissolution. In Chemistry and Prevention of Dental Caries, ed. R. F. SognNaEs, chap. 5, pp. 164-79. Springfield, Ill.: Charles C Thomas, 1962.

5. Gray, J. A., and Francis, M. D. Physical Chemistry of Enamel Dissolution. In Destruction of Hard Tissues, ed. R. F. Sognnaes. Washington, D.C.: American Association for the Advancement of Science, 1963.

6. Rootare, H. M., Deitz, V. R., and Carpenter, F. G. Solubility Product Phenomena in HydroxyapatiteWater Systems, J. Colloid Sci., 17:179-206, 1962.

7. Brown, W. E. Behavior of Slightly Soluble Calcium Phosphates as Revealed by Phase-Equilibrium Calculations, Soil Sci., 90:51-57, 1960.

8. Robinson, R. A., and Stokes, R. H. Electrolyte Solutions, p. 482. London: Academic Press, Inc., 1959.

9. Levinskas, G. J., and Neuman, W. F. Solubility of Bone Mineral. I. Solubility Studies of Synthetic $\mathrm{Hy}-$ droxyapatites, $J$. phys. Chem., 59:164-68, 1955.

10. Strates, B. S., Neuman, W. F., and Levinskas, G. J. The Solubility of Bone Mineral. IT. Precipitation of Near Neutral Solutions of Calcium and Phosphate, $J$. phys. Chem., 61:279-82, 1957.

11. Kielland, J. Individual Activity Coefficients of Ions in Aqueous Solutions, J. Amer. chem. Soc., 59:1675$78,1937$.

12. Jost, W. Diffusion in Solids, Liquids, and Gases, p. 476. New York: Academic Press, Inc., 1960.

13. Glasstone, S. An Introduction to Electrochemistry, p. 448. New York: D. Van Nostrand \& Co., 1942.

14. Cannan, R. K., and Krbrick, A. Complex Formation between Carboxylic Acids and Divalent Metal Cations, J. Amer. Chem. Soc., 60:2314-20, 1938. 